\title{
Editorial
}

\section{Semantic Web - Interoperability, Usability, Applicability}

\author{
Pascal Hitzler ${ }^{\mathrm{a}}$ and Krzysztof Janowicz ${ }^{\mathrm{b}, *}$ \\ ${ }^{a}$ Kno.e.sis Center, Wright State University, USA \\ ${ }^{\mathrm{b}}$ GeoVISTA Center, Pennsylvania State University, USA
}

\section{The Semantic Web is here to stay.}

While this statement seems obvious, this has not been so a few years ago, when basic research funding seemed to be running out, and industrial uptake was hardly happening. In the meantime, we do not only see sustained funding for Semantic Web related research (in particular by the European Commission), but also significant investment by industry, including major IT and venture capital companies. The Semantic Web is here to stay - and to grow.

The Semantic Web is multidisciplinary and heterogeneous. Many Semantic Web researchers maintain close ties to neighboring disciplines which provide methods or application areas for their work. However, the Semantic Web has now established itself as a research field in its own rights. Consequently, a growing number of researchers, in particular those of the second or third generation, seem to identify themselves with the Semantic Web as their primary field of work. The growing number of top quality events dedicated to Semantic Web topics is also a clear indication of this trend. Another indicator is the increasing interweavement of Semantic Web methods into related disciplines leading to research topics such as geospatialsemantics, the Semantic Sensor Web, semantic desktop, or work on cultural heritage.

The Semantic Web journal is set up to be a forum for highest-quality research contributions on all aspects of the Semantic Web. Its scope encompasses work in neighboring disciplines which is motivated by

\footnotetext{
*Corresponding author. E-mail: jano@psu.edu.
}

the Semantic Web vision. Besides the publishing of research contributions, it is also an outlet for reports on tools, systems, applications, and ontologies which enable research, rather than being direct research contributions. ${ }^{1}$ The journal also publishes top-quality surveys which serve as introductions to core topics of Semantic Web research.

The journal's subtitle - Interoperability, Usability, Applicability - reflects the wide scope of the journal, by putting an emphasis on enabling new technologies and methods. Interoperability refers to aspects such as the seamless integration of data from heterogeneous sources, on-the-fly composition and interoperation of Web services, and next-generation search engines. Usability encompasses new information retrieval paradigms, user interfaces and interaction, and visualization techniques, which in turn require methods for dealing with context dependency, personalization, trust, and provenance, amongst others, while hiding the underlying computational issues from the user. Applicability refers to the rapidly growing application areas of Semantic Web technologies and methods, to the issue of bringing state-of-the-art research results to bear on real-world applications, and to the development of new methods and foundations driven by real application needs from various domains.

The primary modern purpose of a scientific journal is quality control and visibility. Fair quality control in scientific publishing directly depends on the quality

${ }^{1}$ See http://www.semantic-web-journal.net/authors/ for information on different types of papers accepted for publication. 
of the underlying review process and further editorial choices. Today, however, reviewing and publishing is inflationary, which increases potential conflicts of interest and substantially reduces the quality of the typical paper - and of the typical review. While we cannot simply reverse this trend, we can take advantage of the World Wide Web to counteract these developments and improve quality and transparency by bringing the review process out into the public space.

The Semantic Web journal thus relies on an open and transparent review process. ${ }^{2}$ All submitted papers as well as the corresponding solicited reviews are made publicly available. All researchers can additionally contribute public reviews and submit comments. Reviewers and editors are publicly known by name. Discussions between reviewers and authors can (and should) happen in public. Reviewers and editors are acknowledged by name in the published versions of the papers.

Reviewers put more effort into providing constructive reviews if their work and contribution to the final manuscript becomes visible. Editors can document their choice of reviewers. Authors can receive additional feedback to ensure that their submission is of sufficient maturity for an archival journal. Public discussions on controversial submissions minimize errors in the decision making and thus result in a fairer procedure.

The success which ensues the setup of the journal is highly encouraging. Until September 2010, we received more than 50 paper submissions; this does not include the vision statement papers contained in this issue. We have several special issues from various domains lined up, some of which have not been publicly announced yet. Researchers have contributed open reviews without us asking them to. So far, there have only been very few occasions where a solicited reviewer has asked to remain anonymous.

This very first issue of the Semantic Web journal contains vision statements by the members of the Editorial Board. While these contributions were essentially invited, they nevertheless underwent the full, open, and transparent review process of the journal in order to improve quality and clarity. Their publication on the journal's webpage already led to comments and open reviews from external researchers. The resulting collection is an impressive compilation of topics of core concern to the Semantic Web community. While it cannot possibly be exhaustive in terms of the many aspects of Semantic Web research, its breadth of coverage is indicative of the breadth of scope of the journal.

We thank all contributors, authors and reviewers alike. If you would also like to contribute to the journal in any way, you can find us at http://www.semanticweb-journal.net/.

\section{Acknowledgements}

This journal wouldn't be here without Arnoud de Kemp. We thank him for taking the initiative. This journal wouldn't be sustainable without all the hard work put into it by the editorial board members, and by the responsible staff at IOS Press. Thank you for your support.

${ }^{2}$ See http://www.semantic-web-journal.net/reviewers\#review. 